\title{
REVIEW
}

\section{Functional genomics of human brain development and implications for autism spectrum disorders}

\author{
MN Ziats $^{1,2,3}$, LP Grosvenor ${ }^{4}$ and OM Rennert ${ }^{1}$
}

Transcription of the inherited DNA sequence into copies of messenger RNA is the most fundamental process by which the genome functions to guide development. Encoded sequence information, inherited epigenetic marks and environmental influences all converge at the level of mRNA gene expression to allow for cell-type-specific, tissue-specific, spatial and temporal patterns of expression. Thus, the transcriptome represents a complex interplay between inherited genomic structure, dynamic experiential demands and external signals. This property makes transcriptome studies uniquely positioned to provide insight into complex genetic-epigenetic-environmental processes such as human brain development, and disorders with non-Mendelian genetic etiologies such as autism spectrum disorders. In this review, we describe recent studies exploring the unique functional genomics profile of the human brain during neurodevelopment. We then highlight two emerging areas of research with great potential to increase our understanding of functional neurogenomics-non-coding RNA expression and gene interaction networks. Finally, we review previous functional genomics studies of autism spectrum disorder in this context, and discuss how investigations at the level of functional genomics are beginning to identify convergent molecular mechanisms underlying this genetically heterogeneous disorder.

Translational Psychiatry (2015) 5, e665; doi:10.1038/tp.2015.153; published online 27 October 2015

\section{HUMAN BRAIN GENE EXPRESSION}

Across human development, a single gene can be expressed in multiple isoforms and varying orders of magnitude depending on the particular developmental context; that is, the tissue, stage of development and local or long-distance signaling mechanisms being received by the cell or tissue. Therefore, in order to understand how a gene may contribute to a developmental disorder, it is critical to first understand its normal expression pattern and function in the appropriate tissue during the developmental time window of interest. This is especially important for human neurodevelopment, as human brain gene expression in particular has been demonstrated to be unique in a number of ways (Figure 1). The consistent reports describing the uniqueness of the human neurodevelopmental transcriptome underscore the need to study genes implicated in neurodevelopmental disorders in their appropriate functional genomic context, in order to understand their role in disease more accurately.

Compared with other species, human brains express messenger RNA (mRNA) transcripts at much higher levels and with much greater complexity. For instance, comparisons of human brain gene expression with both mice ${ }^{1,2}$ and primates ${ }^{3,4}$ has demonstrated that most of the differentially expressed genes between the species are upregulated in humans, but this phenomena is not apparent in other tissues. In addition, the human brain expresses $\sim 85 \%$ of all genes encoded in the human genome at some point during development, ${ }^{5}$ which is greater than any other human tissue type. It is hypothesized that this increased level of gene expression is at least partially responsible for the higher level of neuronal activity and overall cognitive function of humans.

Within humans specifically, the brain also displays a distinct gene expression profile compared with other tissues. Using both array $^{6}$ and sequencing-based techniques, ${ }^{7}$ the brain has been shown to have higher expression levels and greater transcriptome complexity than most other human tissue and cell and tissue types, with perhaps the notable exception of sperm. ${ }^{8,9}$ In particular, the human brain transcriptome displays a high level of alternatively spliced transcripts, ${ }^{10-12}$ and the set of isoforms produced in brain differs considerably from other tissue types. ${ }^{6,10}$ In addition, the human brain transcriptome also contains a distinctively high number of non-coding RNAs (ncRNAs). In fact, the brain displays the greatest abundance of transcribed ncRNAs among all tissues. ${ }^{13}$ Both short ncRNAs, such as microRNAs (miRNAs) and PIWl-interacting RNAs, and long ncRNAs (IncRNAs) are highly enriched in human brain tissue. ${ }^{14-18}$ As ncRNAs are becoming increasingly recognized as important regulatory elements in genome processing during neurodevelopment, and in the pathogenesis of neurodevelopmental disorders, ${ }^{19}$ their abundance in the brain further highlights the uniqueness of neurodevelopmental functional genomics.

Although within a given brain region the human transcriptome has been shown to be incredibly complex, it is also of importance to consider the relationship among different anatomical regions of the brain, as 'disconnectivity' between disparate brain regions is thought to underlie a number of neurodevelopmental syndromes such as autism spectrum disorders (ASD). ${ }^{20,21}$ Perhaps

\footnotetext{
${ }^{1}$ Laboratory of Clinical and Developmental Genomics, National Institute of Child Health and Human Development, National Institutes of Health, Bethesda, MD, USA; ${ }^{2}$ University of Cambridge, Robinson College, Cambridgeshire, UK; ${ }^{3}$ Baylor College of Medicine MSTP, One Baylor Plaza, Houston, TX, USA and ${ }^{4}$ Pediatrics and Developmental Neuroscience Branch, National Institute of Mental Health, National Institutes of Health, Bethesda, MD, USA. Correspondence: Dr MN Ziats, Laboratory of Clinical and Developmental Genomics, National Institute of Child Health and Human Development, National Institutes of Health, 49 Convent Drive, Building 49, Room 2C08, Bethesda, MD 20814, USA.

E-mail: ziats@bcm.edu
}

Received 27 February 2014; revised 3 September 2015; accepted 6 September 2015 


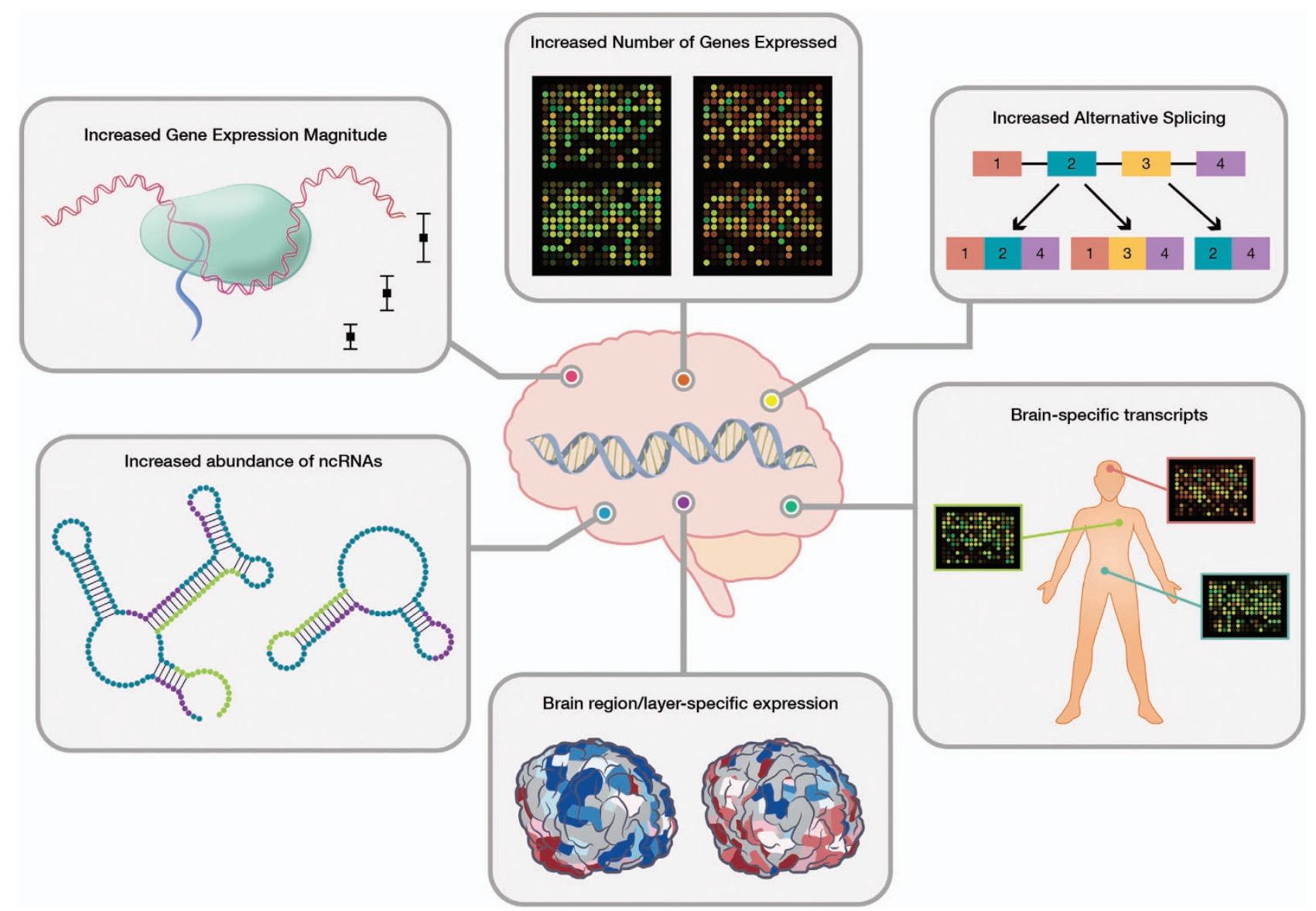

Figure 1. Summary of unique functional genomic properties of the human brain. Unique human-specific RNA expression properties discussed in the text are diagrammed. ncRNA, non-coding RNA.

unsurprisingly, there is strong evidence that distinct regions of the human brain have distinct gene expression profiles, and animal studies have suggested that this variation is related to both structural and functional differences. ${ }^{19}$ For instance, a microarray study of 20 distinct brain and spinal cord sites showed that expression profiles can cluster samples from different donors by anatomical origin, and that some anatomical regions have up to 2000 region-specific genes. ${ }^{22}$ Multiple studies have shown that the cerebellum contains the most unique gene expression pattern compared with other brain structures, $2,22,23$ which is of consequence to the neurodevelopmental disorder autism in particular, as this region has been consistently implicated in its pathogenesis. ${ }^{24}$ Even within the neocortex alone, different cortical layers each express a distinct profile of mRNA transcripts. ${ }^{25}$ Further highlighting the importance of these region- and layer-specific expression properties are a number of reports that have shown that gene expression differences between any two brain regions within one individual are more pronounced than are gene expression differences between the same brain region of two different individuals. ${ }^{4,23,26}$

Taken together, these findings highlight the importance of understanding human psychiatric and neurodevelopmental disorders in the context of human brain gene expression specifically, as it is possible that animal, cellular and other models do not recapitulate human brain mRNA and ncRNA expression patterns with the appropriate level of fidelity. Although animal and cellular studies have and will continue to play an invaluable role in understanding how ncRNAs mechanistically influence gene expression and other processes at the molecular level, the identification of ncRNAs of relevance to neurodevelopmental disorders is most likely to come from post-mortem brain tissue studies of these diseases. Moreover, evidence is accumulating that suggests gene expression patterns within the human brain vary considerably across developmental time, and therefore temporal patterns of gene expression are an important consideration for neurodevelopmental disorders with childhood onset.

\section{CHANGES IN GENE EXPRESSION DURING HUMAN NEURODEVELOPMENT}

The developing human brain grows remarkably fast-the weight of a newborn's brain is $\sim 25 \%$ of its adult weight, but within 2 years, it nearly reaches adult size. ${ }^{27}$ During this time, the brain grows mainly through glial multiplication, myelination, formation of new synaptic connections and pruning of unused synaptic connections. Although the human brain continues to mature up to the age of 25,28 the greatest changes occur during infancy and early childhood. Coincidentally, most neurodevelopmental disorders become clinically recognizable around these ages.

Underlying these marked early changes in gross brain development are complex and dynamic broad patterns of gene expression change, which have only recently begun to be understood. The most comprehensive study to date of the developing human brain transcriptome documented that genome-wide patterns of expression correspond closely to the major stages of clinical development (namely prenatal, early infancy, childhood, adolescence and adulthood), and that the molecular profile of these stages are distinct from each other. ${ }^{5}$ The most striking observation was that the greatest shift in gene expression occurs around the period of birth, when almost $60 \%$ of 
genes change their expression patterns in the neocortex. Other studies have demonstrated similar changes, and have shown that many of the genes identified during this shift are involved in cortical development and higher-order cognitive functioning. ${ }^{29,30}$

The microarray study by Kang et al., ${ }^{5}$ which assessed neurologically normal donor brains spanning the 2nd trimester through adulthood, also demonstrated that after infancy the number of genes whose expression profile changes in the neocortex decreases markedly to $\sim 9 \%$ of expressed genes between infancy and adolescence, and less than $1 \%$ of genes between adolescence and adulthood. Functional annotation of these gene sets further revealed that genes expressed very early in prenatal development are highly related to the processes of cell differentiation, proliferation and migration, whereas genes expressed later in gestation are more related to synaptogenesis -suggesting that time-period-specific gene expression patterns drive cell-level developmental programs. Again, these findings highlight the importance of assessing candidate disease genes during the appropriate developmental time window, in order to gain the most relevant insight into their neurodevelopmental functions.

In addition to the greatest number of genes shifting their expression trajectory shortly after birth, the changes in gene expression in early postnatal life also have greater amplitudes of change than during other times. ${ }^{31-33}$ In fact, it was shown that many genes actually completely reverse their expression trajectory in early life, mostly shifting from a trend of increasing expression in fetal life and infancy to decreasing expression beginning in childhood. ${ }^{31}$ Moreover, as the brain begins to mature, the gene expression profile within each anatomical region becomes more similar to other brain regions (with the notable exception of the cerebellum), suggesting that most of the genetically encoded region-specific development is completed early in life. Interestingly, these broad gene expression patterns appear to reverse themselves in older age, at least in the prefrontal cortex. ${ }^{33}$

\section{NON-CODING RNAs IN HUMAN BRAIN DEVELOPMENT}

Since the advent of high-throughput, unbiased, genome-wide expression arrays and sequencing platforms, the recognition that the genome is pervasively transcribed at loci that do not encode for protein has become well recognized. Although originally considered to represent evolutionary 'noise,' the non-coding component of the transcriptome has been increasingly shown to regulate the genomic landscape though a myriad of mechanisms, and as such are increasingly being recognized as important modulators of gene expression. ${ }^{34}$ Likewise, they are also beginning to be implicated in neurological disease.

NcRNAs can modulate transcription in a number of ways, such as interacting with DNA to induce methylation or histone modifications, recruiting transcription factors to promoters and modulating the three-dimensional architecture of chromosomes in the nucleus. ${ }^{35,36}$ They can also bind to other RNA molecules, especially mRNAs with complementary sequences, to inhibit translation through RNA degradation, or they can act as 'sponges' and thereby dilute the effect of mRNAs or other ncRNAs. ${ }^{37}$ Conversely, they can increase the rate of translation by acting as molecular stabilizing scaffolds, catalyze protein-protein interactions by linking otherwise scarce proteins together and participate in cellular trafficking of RNA-binding proteins. ${ }^{38}$ In addition, ncRNAs have even been shown to participate in intercellular communication by helping transport cargo between adjacent cells. ${ }^{39,40}$

The increasing recognition of this important layer of transcriptome information is perhaps most important in the brain, where it has been shown that IncRNAs in particular are abundantly expressed in brain tissue, ${ }^{41}$ and that greatest abundance of
ncRNAs appears to exist in brain (and perhaps testis) as compared with other tissue types. ${ }^{42,43}$ Furthermore, there is strong evidence that ncRNAs had a critical role in the evolution of human brain structure and function. For instance, the fastest evolving regions of the primate genome are sequences that are transcribed into ncRNAs, and it has been shown that these particular ncRNAs are primarily involved in regulating neurodevelopmental genes. ${ }^{44}$ Similarly, regulation of transcription through RNA editing (which is particularly frequent among ncRNAs) has undergone a significant evolutionary expansion in higher primates and humans. ${ }^{45}$

This expanding inventory of ncRNAs, and their increasing functional and regulatory activities in humans, appear to have an important role in neurodevelopment and neuropsychiatric diseases. In particular, miRNAs and IncRNAs are the two most understood types of regulatory RNAs, and have recently been implicated in a number of neurodevelopmental disorders including ASD.

\section{MICRORNAs}

The best studied of the ncRNAs are miRNAs, which mainly function to repress translation post-transcriptionally through the RNA interference mechanism. The miRNA family includes a variety of precursor RNA molecules that are classified mainly on their genomic origin, such as endogenous small interfering RNAs and PIWI-interacting RNAs. Despite their origin, all classes of small RNAs are quickly processed after transcription into their mature form, which for miRNAs is typically a 20-23-nucleotide, singlestranded molecule. ${ }^{46}$

In brief, the RNA interference mechanism begins when precursor miRNAs are transcribed in their entirety from the genomic DNA, processed by an enzyme complex known as DROSHA, exported to the cytoplasm and cleaved by the ribonuclease DICER into their mature form. At this point, they bind to a class of proteins termed Argonautes, and are then incorporated into a larger multi-protein complex termed the RNAinduced silence complex. The RNA-induced silence complex is guided to mRNAs that are complementary to the associated miRNA, leading to a repression of translation or overt degradation of the mRNA transcript. ${ }^{47}$

Importantly, a single miRNA can target-and therefore regulate -many mRNAs because of their short sequence, their preferential binding to $3^{\prime}$-untranslated regions and their imperfect complementary binding to cognate sequences. ${ }^{48}$ Conversely, it has been shown that individual mRNAs are often targeted by multiple miRNAs. Therefore, miRNA-mRNA interactions alone can increase the complexity of gene expression regulation by orders of magnitude. Furthermore, miRNAs are known to target other ncRNAs in addition to protein-coding mRNAs. ${ }^{49}$ The critical implication of these insights is that a single miRNA has the ability to modulate entire transcriptional networks, and therefore the mis-expression of a single miRNA has the potential to disrupt the proper expression of entire suites of genes.

Recent studies have demonstrated the importance of miRNAs in human brain evolution, cellular development, experiencedependent plasticity and in neuropsychiatric disorders. A large number of miRNAs exhibit species-specific expression patterns, are conserved only in primates and/or humans or are exclusively expressed in brain-providing strong evidence for their role in human-specific brain functions and disorders. ${ }^{33,50}$ For example, an analysis of human, chimpanzee and macaque prefrontal cortex and cerebellum showed a substantial degree of divergence in their miRNA expression patterns. ${ }^{51}$

In addition, important roles for miRNAs in neural stem cell maintenance and differentiation have been established through a number of studies that have identified and characterized individual miRNAs of interest. For instance, multiple studies have shown that DICER knockout animals display a host of 
neurodevelopmental defects, including abnormal brain size, structural defects and improper formation of synapses. ${ }^{52-54}$ One particular miRNA, miR-9, has been studied extensively for its role in developmental patterning and cell migration, where it has been shown to be critical for neural stem cell self-renewal, ${ }^{55}$ production of some of the earliest neurons in the developing telencephalon and cortical laminization. ${ }^{56}$ Cellular studies of pluripotency have demonstrated that introduction of particular miRNAs can reprogram human skin fibroblasts into neuronal-like cells, ${ }^{57}$ and that this mechanism likely involves the central nature of these miRNAs in canonical transcriptional networks, which are known to guide neural cell fate decisions. ${ }^{58}$ In addition to neurogenesis, miRNAs have also been shown to regulate gliogenesis, in particular the formation of oligodendrocytes and astrocytes..$^{59,60}$

In addition to their role in individual cellular-level functions, miRNAs have been shown to contribute to synaptogenesis and experience-dependent plasticity-both functions thought to underlie complex human behavior, and hypothesized to be disrupted in ASD. For example, specific miRNAs are enriched in the synaptic nerve terminals of axons, ${ }^{61}$ and are upregulated in expression in the hippocampus following memory tasks in mice. ${ }^{62}$ In Drosophila melanogaster, knockout of subcomponents of the DICER complex results in synaptic transmission defects, but no overt brain structural abnormalities, ${ }^{63}$ and in mice result in dendritic spine malformations ${ }^{53}$ and impaired synaptic transmission. ${ }^{64}$ Remarkably, a complete absence of mature miRNAs due to DICER knockout affects memory and learning in mice. ${ }^{65}$

Finally, miRNAs have recently been recognized as contributing to human neurologic disease. Inherited variation in DNA encoding for miRNAs or their recognition sites has been linked to a number of disorders including schizophrenia. ${ }^{66}$ Dysregulated expression of miRNAs has been demonstrated in brain tumors, ${ }^{67}$ Parkinson's disease $^{68}$ and Tourette's syndrome. ${ }^{69}$ Expression of miRNAs in normal human developing brain appears to be highly brain-region-, developmental-stage-, and even sex specific, and the putative target genes of the differentially expressed miRNAs were found to be highly related to known neurodevelopmental disorder risk genes. ${ }^{70}$

However, despite these substantial observations suggesting that miRNAs are critical regulators of neurodevelopmental transcriptional networks and are often disrupted in neurologic diseases, only a few small studies have attempted to profile miRNA expression levels in neurodevelopmental disorders such as ASD (reviewed below). Thus, there is a need for a comprehensive assessment of miRNAs in post-mortem brain tissue from individuals affected by neurodevelopmental disorders.

\section{LONG NON-CODING RNAs}

In contrast to miRNAs, which are short sequences with welldefined functions in post-transcriptional regulation, IncRNAs represent a novel class of transcripts whose function in brain development remains poorly understood. LncRNAs are defined as RNAs greater than 200 nucleotides in length (as compared with 21-23 nucleotide length of miRNAs), which do not encode for protein, or lack an appreciable reading frame. LncRNAs undergo post-transcriptional processing similar to other RNAs, providing the first hint at their functional importance. For instance, some IncRNAs are modified to include a 5'-methyl cap and undergo 3'polyadenlyation. ${ }^{71,72}$ However, unlike miRNAs, IncRNAs are generally poorly conserved evolutionarily and as such, elucidation of their functional roles has relied more upon expression analysis and individual functional studies than on comparative genomic interpretations.

Although originally thought to be evolutionary byproducts of 'junk DNA,' IncRNAs have been shown to be involved in major mechanisms of gene expression regulation, such as targeting transcription factors, initiating chromatin remodeling, directing methylation complexes and blocking nearby transcription. ${ }^{35}$ Moreover, pervasive transcription of IncRNAs has been demonstrated to occur in both a temporally and spatially regulated manner during development, ${ }^{73}$ with the central nervous system displaying the greatest abundance of transcribed IncRNAs. ${ }^{41-43}$

Recently, it has been demonstrated that individual IncRNAs have important regulatory roles in the spatial-temporal control of gene expression in the brain. One of the first studies to demonstrate this explored RNA expression from mouse in situ hybridization data, and the authors demonstrated that most IncRNAs examined were localized to specific cell types, subcellular compartments or neuroanatomical regions. ${ }^{41}$ This work provided some of the first large-scale evidence that IncRNAs may have specific functions in their capacity as RNAs alone in the mammalian brain. Subsequently, a number of studies employing both whole-genome and individual candidate IncRNA assessment have begun to expose the importance of IncRNAs to the regulation of the developing brain transcriptome. For example, individual IncRNAs are upregulated in response to neural activity and synaptogenesis. ${ }^{74,75}$ They have also been implicated in neuronal differentiation; for instance, the IncRNA Evf2 recruits a number of important neurodevelopmental transcription factors (such as Mecp2, the DLX family and GAD1) to their target genes in GABA-ergic interneurons, and Evf2 knockout mice have reduced interneuron cell numbers. ${ }^{76}$ Transcriptome studies of brain tissue have also begun to characterize the entire landscape of IncRNAs during neurodevelopment. It was shown that IncRNAs are differentially expressed across layers of the mouse neocortex, ${ }^{77}$ and that those expressed in brain are preferentially located in genomic regions containing critical neurodevelopmental genes. ${ }^{16}$

LncRNAs have also been found to be abnormally expressed in a number of neurologic disorders. The first rigorous demonstration of the mechanistic function of a IncRNA in human neuronal activity and subsequent dysfunctional mechanism in disease was recently described for the IncRNA Gomafu, where the authors showed that Gomafu binds directly to the splicing factors QKI and SRSF1, and dysregulation of Gomafu expression leads to alternative splicing patterns that resemble those observed in SZ post-mortem brain tissue. ${ }^{78}$ Another example is the IncRNA BACE1-AS. This IncRNA is an antisense transcript of the beta-secretase- 1 gene locus, which is implicated in the generation of beta-amyloid plaques in Alzheimer's disease. ${ }^{79}$ The BACE1-AS IncRNA extensively regulates the level of $B A C E 1$, and therefore can directly affect the level of beta-amyloid plaque accumulation. ${ }^{80}$ Other IncRNAs have been implicated in the neurodevelopmental disorder Angelman's syndrome, which shares many features with ASD. ${ }^{81,82}$

Therefore, IncRNAs appear to have a critical role in modulating gene expression in the developing brain, and are increasingly implicated in neurological disorders. Their potentially numerous regulatory mechanisms, and largely overlooked sequence variation in disease, makes them an important class of candidate molecules to consider in neurodevelopmental disorders with complex genetics, such as ASD.

\section{GENE NETWORKS IN THE DEVELOPING HUMAN BRAIN}

Although assessing non-coding regions of the genome is an important approach to comprehensively understanding the complex functional genomics of human brain development and neurodevelopmental disorders, it is equally important to consider how disparate genomic elements may work in concert with each other to produce biological effects that are emergent only after their interaction. The study of genetic interactions can be done by modeling large gene sets as networks of interacting nodes and edges, allowing for a statistical assessment of relationships among and between genes, as opposed to the study of individual genes themselves. Such approaches are particularly important in complex genetic syndromes such as ASD, as genome-wide 

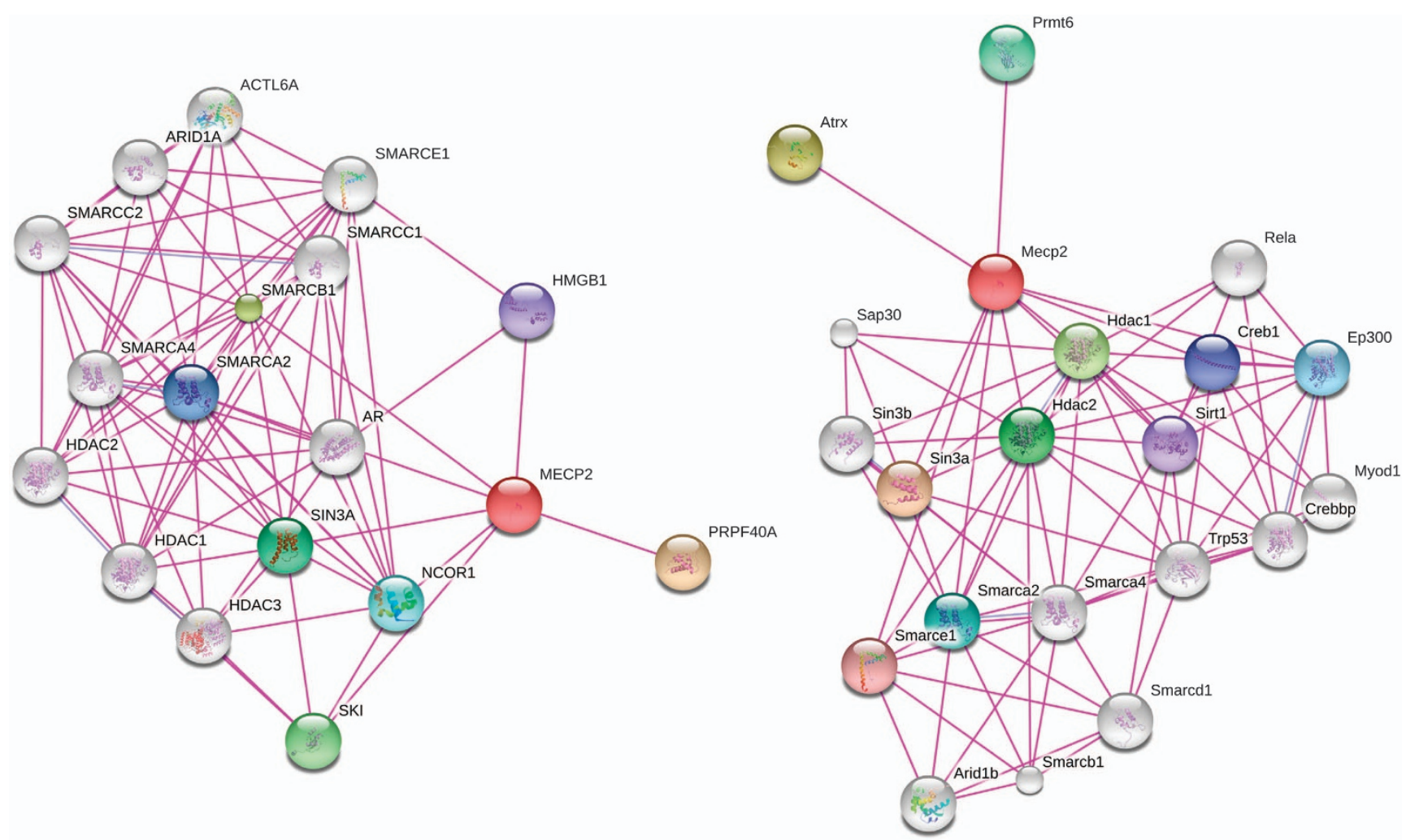

Figure 2. Examples of a protein interaction networks. The properties of networks as a whole can become apparent, which would not be appreciated by studying individual genes. For example, the network on the left represents known 2nd-degree protein-protein interactions with the gene Mecp2 in humans, whereas the network on the right represents the known Mecp2 interactions in mice. As can be seen, the human network (left) is much more densely connected, suggesting Mecp2 has more known interactions in humans. More rigorous methods are available to quantify the degree of connectedness of such networks. Networks in this figure were created with String v9.05 (http://stringdb.org/).

association studies have consistently demonstrated that most individual variants in ASD have only very small effects by themselves.

The study of gene networks is a subset of the field of network science that applies mathematical and statistical principles to biological systems. A biological network is a system of individual biologic components that interact with each other in a structured, non-random manner, such that properties of the network as a whole emerge that are not apparent by studying the individual components in isolation. Biological networks have been identified at levels spanning molecular, ${ }^{83}$ cellular, ${ }^{84}$ organ system ${ }^{85}$ and even inter-individual relationships. ${ }^{86}$

An interaction network consists of nodes and edges. In gene networks, nodes represent discrete genes and edges represent a biological relationship or connection between nodes (Figure 2). Depending on the interaction being studied, edges can represent many relationships such as known protein-protein binding, correlations of expression levels between genes, or any other metric that can be measured in all nodes and have putative biological relevance to how the system works. As most gene networks are incredibly large (thousands of nodes and millions of possible edges), the study of biological networks relies on a number of mathematical principles adopted from network theory and statistics that allows for the incredible complexity of large networks to be summarized, quantified and visualized in order to more easily infer biological meaning.

A genetic interaction occurs when an unexpected phenotype emerges from the combination of two or more interacting genes. This phenomenon is widely pervasive in genetics and has been recognized for decades. For instance, the phenomenon of synthetic lethality occurs when two genetic mutations, which by themselves cause no effect, are both present in the same gene and their presence together results in a defective protein product. $^{87}$ Furthermore, genetic interactions appear to be ubiquitous throughout the genome. ${ }^{88}$ However, predicting how independent genes combine with each other to create emergent properties is not straightforward. This is especially true in nonmodel organism systems, such as the human brain, where it is impossible to manipulate individual genes, and therefore researchers must rely only on observational measures such as gene and protein expression levels.

One validated approach to integrate heterogeneous gene sets, in order to uncover shared molecular mechanisms, is through the analysis of gene co-expression patterns, which invoke the guilt-byassociation heuristic that is pervasive in genomics research. ${ }^{89,90}$ Several studies have demonstrated that genes with similar brain co-expression patterns are likely to function together in common cellular pathways. ${ }^{91,92}$ These transcriptional co-expression relationships are particularly relevant to neurodevelopment, as the precise regulation of gene expression across brain regions at different ages instructs the exquisite specialization and connectivity within the brain. For instance, if two genes are expressed with similar patterns (that is, they have a similar magnitude and direction of expression change across developmental time), they would have a higher correlation than two genes whose expression appears to be randomly related to one another. In this network, edges would link genes with similar expression profiles, whereas unrelated genes would not share an edge. Defining edges between genes in this way allows the conclusion that the two nodes share related biological function, and can be used to derive and study large-scale genetic interaction networks. 
Another widely used approach to infer interaction networks is to draw upon experimentally determined protein-protein interactions. Protein-protein interaction networks are perhaps the best-characterized networks in all of biology, and many wellcurated experimental data sets containing detailed interaction information exist. Studies have demonstrated that protein interaction networks are conserved evolutionarily, ${ }^{93}$ and that proteins in the network with high degrees of connectedness are more important for organismal survival and fitness than are networks with lesser connectivity. ${ }^{94}$ This suggests that information on the importance of individual genes/proteins in a network can be inferred by studying the overall structure of the network as a whole. Such an approach could be particularly valuable in disorders such as ASD, where there are many implicated genes, but the relative importance of each to the pathophysiology of the disorder is unclear.

As neurodevelopmental disorders such as autism are believed to result from functional aberrations within brain regions and/or disruption of inter-regional connectivity between regions, ${ }^{20}$ investigating the gene expression profiles of autism candidate genes across brain regions and throughout normal human neurodevelopment may provide insight into the complex functional genomics of this neurodevelopmental disorder. Furthermore, as the genetic heterogeneity of ASD continues to increase as more sequencing and association studies are performed, prioritizing candidate genes through their location in interaction networks is an important undertaking.

\section{FUNCTIONAL GENOMICS STUDIES OF ASD}

Gene expression dynamics in early human brain development are clearly both spatially and temporally specific. This suggests not only that they are highly regulated, but that different genes and gene networks will have dynamic expression throughout space and time. Despite this increasingly recognized property of human neurodevelopmental genomics, until recently few studies of autism candidate genes have considered their expression and function in early human brain development. Furthermore, the molecular regulators of brain mRNA expression, such as ncRNAs, have not been extensively characterized in the developing human brain, and their potential involvement in ASD is only beginning to be explored. Accordingly, an understanding of the functional genomics of autism, the genetic interaction networks that ASD candidate genes participate in and the potential ncRNA regulators of these genes, represent important unresolved avenues of research.

\section{GENE EXPRESSION STUDIES IN ASD}

The majority of gene expression studies in autism have been performed in peripheral blood lymphocytes; ${ }^{95}$ however, as only approximately half of the genes expressed in brain are also expressed in lymphocytes, ${ }^{96}$ it is critically important that autistic brain tissue be assessed directly. The few genome-wide expression profiling studies in autistic brain tissue have repeatedly identified a number of functions that appear to be disrupted in autistic brain. The first microarray study of autistic brain tissue assessed post-mortem cerebellum and demonstrated dysregulation of AMPA receptor subunits in ASD. ${ }^{97}$ Subsequently, Garbett et al. ${ }^{98}$ analyzed genome-wide microarray expression profiles from six autistic temporal cortices and six controls, and their results suggested an upregulation of genes involved in immune and inflammatory processes with a concurrent downregulation of genes involved in neurodevelopment. The largest sample size assessed by microarray analysis to date studied three separate brain regions (frontal cortex, temporal cortex and cerebellum) from 19 autistic cases and 17 controls. ${ }^{99}$ This work also demonstrated an upregulation of genes with known immune function and a concurrent downregulation of genes involved with the synapse. Importantly, there was a large degree of overlap in the genes identified between the studies by Garbett et al. ${ }^{98}$ and Voineagu et al., ${ }^{99}$ even though they assessed different cohorts of donor brains. More recently, a study of the autistic prefrontal cortex across a large age span demonstrated dysregulation in pathways governing cell number, cortical patterning and differentiation in the young autistic prefrontal cortex, but in contrast found dysregulation of signaling and repair pathways in adult autistic brain tissue. ${ }^{100}$ Another recent study of autistic cerebellar and occipital brain regions demonstrated significant gene expression abnormalities in mitochondrial oxidative phosphorylation and protein translation pathways. ${ }^{101}$

Although the number of studies assessing gene expression in autistic brain are still small, already there appears to be a growing body of evidence implicating disrupted molecular pathways involved in synaptogenesis and immune function, in addition to a number of molecular mechanisms such as transcription and translation. However, how these observations relate to known inherited mutations in autistic candidate genes, or how disruptions of candidate gene's expression may result in these broad molecular changes, is far from clear.

\section{NON-CODING RNA STUDIES IN ASD}

Remarkably, most of the single-nucleotide polymorphisms associated with ASD in genome-wide association studies have been in intergenic regions or intronic sequences outside the proteincoding DNA. ${ }^{102}$ Despite these results and the increasing recognition of the importance of ncRNAs in the molecular regulation of human brain development, assessment of ncRNAs in ASD has been limited. Moreover, despite the known tissue-specific nature of ncRNA expression, few of the ncRNA studies in autistic samples have been performed on brain tissue specifically.

To date, four studies have profiled miRNA expression in tissues derived from patients with 'idiopathic' autism. In the only study of autistic post-mortem brain tissue, Abu-Elneel et al. ${ }^{103}$ identified 28 differentially expressed miRNAs in ASD cerebellum via quantitative reverse transcription-PCR analysis. A number of microarray studies have assessed for miRNA expression differences in blood lymphocytes of autistic patients as compared with controls, having discovered nearly 100 miRNAs that are abnormally expressed in ASD. ${ }^{104-106}$ Another study recently described aberrant expression of IncRNAs in autistic frontal cortex and cerebellum that are antisense transcripts to known autism candidate genes. ${ }^{107}$ This work supports a small study showing IncRNAs are differentially expressed in ASD post-mortem prefrontal cortex and cerebellum, and that previously reported patterning deficits of the mRNA component of the transcriptome in autistic brain are also apparent among IncRNAs. ${ }^{108}$

In addition, recent work has shown that at least $40 \%$ of loci previously implicated in ASD abundantly express non-coding antisense transcripts, that these transcripts are often expressed in brain-region-specific manners during development and some are abnormally expressed in autistic post-mortem brain tissue. ${ }^{107}$ Similarly, an earlier study identified a highly significant singlenucleotide polymorphism enriched among ASD patients through genome-wide association analysis, and found it resides in a gene poor locus on chromosome $5 \mathrm{p} 14.1$. Further analysis demonstrated that this locus produces an antisense IncRNA to a pseudogene of the mRNA Moesin, and that this IncRNA binds to Moesin in cells, can alter its expression and that Moesin is significantly differentially expressed in post-mortem brain tissue from autistic patients. ${ }^{109}$ Important studies such as these are beginning to link variations found at the DNA sequence level to ncRNA expression differences demonstrated in post-mortem autistic brain tissue.

Therefore, although it appears that ncRNAs are abnormal in ASD patients, their expression and function during human brain 
development has not been thoroughly characterized, and apart from a few studies attempting to identify mis-expressed ncRNAs, no work has functionally demonstrated how ncRNAs may ultimately contribute to the ASD phenotype. Consequently, there is a critical need to assess for changes in miRNA and IncRNA expression in autistic brain tissue more thoroughly, and to attempt to determine how these changes affect the development of ASD.

\section{NETWORK AND PATHWAY STUDIES IN ASD}

Finally, several pathway analyses have been performed using either genetic or transcriptome data to gain insight into the biological functions associated with ASD candidate genes. For instance, O'Roak et al. ${ }^{110}$ analyzed protein interaction networks among genes implicated in ASD via whole-exome sequencing studies, and identified that de novo mutations in ASD patients are over-represented among proteins involved in a chromatinremodeling network and the $\beta$-catenin pathway. Similarly, Gilman et al. ${ }^{111}$ demonstrated that copy number variants identified in autistic patients are enriched for genes involved in a molecular network related to synaptogenesis, axon guidance and neuronal motility. These studies represent some of the most rigorous autism cohort sequencing studies performed to date, and their identification of epigenetic processes related to mRNA and ncRNA expression further highlights the importance of this area of research in understanding the molecular mechanisms underlying ASD. ${ }^{103}$

A number of recent studies have attempted to explore the potential functional consequences of autism candidate genes by integrating them with neurodevelopmental gene expression. ${ }^{112}$ Ben-David and Shifman ${ }^{113}$ attempted to assess for differences between rare and common ASD candidate genes by studying their co-expression relationships in adult human brain. They discovered both sets of ASD candidate genes were related to synaptogenesis and neuronal plasticity, and that they are expressed in areas associated with learning, memory and sensory perception. ${ }^{112}$ The same authors also analyzed the neurodevelopmental expression of ASD candidate genes that had been discovered as de novo mutations, and demonstrated that these genes relate to molecular networks involved in transcriptional regulation and chromatin remodeling. ${ }^{113}$

Using the same neurodevelopmental transcriptional data set, two other complementary reports recently attempted to infer common biology among various subsets of ASD candidate genes. In the first study, the authors assessed the co-expression relationships among ASD candidate genes they determined to be 'high confidence' candidates. They demonstrated that these genes are specifically highly co-expressed during mid-fetal development, and in layer $5 / 6$ cortical projection neurons. ${ }^{114}$ The companion study assessed a broader list of ASD candidates, and demonstrated a convergence upon pathways involved in transcriptional regulation during early development, and in synaptogenesis during later childhood. ${ }^{115}$ Although these studies rely on correlative measures to arrive at their conclusions, they provide some of the first rigorous evidence that ASD candidate genes may relate to each other through shared gene expression modules.

\section{CONCLUSION AND FUTURE DIRECTIONS}

The human brain transcriptome displays a remarkably complex array of mRNAs and ncRNAs that are both temporally and spatially specific. Furthermore, comparative genomics studies have shown that the functional genomics of human brain development diverges significantly from model organisms or even close evolutionary relatives. NcRNAs such as miRNAs and IncRNAs are increasingly recognized as critical regulators of gene expression, and the analysis of gene interaction networks allows for the identification of emergent properties among large sets of genes and ncRNAs that are often not apparent when studying individual genes of interest in isolation. Therefore, it is critical that the genes implicated in autism be understood in the specific context of human neurodevelopmental gene expression, that potential critical ncRNA regulators of their expression be identified and that their network-level properties be explored, as the appropriate temporal-spatial-regulatory context is likely very important to complex neurodevelopmental disorders.

Although studies of ncRNAs in human brain development and autism hold much promise for significantly advancing the understanding of these complex genetic-epigenetic-environmental processes, the field as a whole is still very much in its infancy and there remains a tremendous amount of research to be undertaken before a comprehensive understanding of how ncRNAs help modulate brain development and influence neurodevelopmental disorders can be attained. A number of exciting new areas of research should help to quickly advance this work in the near future, notably through assessment of in vitro cell and tissue systems that accurately mimic human brain functional genomics, improved bioinformatics techniques for characterizing ncRNAs and large-scale DNA-sequencing studies of the noncoding regions of the genome in patients with neurodevelopmental disorders.

Foremost, efforts to address the shortage and difficulty of obtaining post-mortem human brain tissue to study ncRNAs are desperately needed if the field is to make significant progress, given the unique human brain-specific patterns of expression previously discussed. ${ }^{116}$ New approaches to modeling human brain development in vitro are emerging that may significantly advance this effort, namely from creating neuron-like cells from human tissue as with induced pluripotent stem cell (iPSC) technology. For instance, two recent studies demonstrated that both human embryonic stem cells and murine neural progenitor cells, which were differentiated into neurons, displayed gene expression profiles that were representative of post-mortem human brain tissue. ${ }^{117}$ In addition, iPSCs have been generated from patients with monogenic neurodevelopmental syndromes such as Angelman and Fragile $X$, potentially allowing for the assessment of disease-specific patterns of ncRNA expression. ${ }^{118}$ Although the use of iPSCs presents advantages for studying native human tissue, there remains concern that the expression and epigenetic signatures of the derived neuron-like cells will more closely mimic the native cell lineage, necessitating continued improvement and expansion of such approaches. ${ }^{119,120}$ One potential emerging method is the generation of 'cerebral organoids,' which use stem cell technology to create threedimesional culture systems that more closely resemble native brain tissue. ${ }^{121}$ Future efforts such as these to advance in vitro model systems to more closely mimic human brain tissue expression patterns will be critical to advance the field.

Finally, the emergence of more sophisticated bioinformatics tools to move from simply cataloging to analyzing the vast and complicated 'ncRNA-ome' is a future step that will be necessary to better interpret signal from noise. One emerging area of research is RNA structure prediction analysis, which can be used to investigate the structure-function relationships of both ncRNAs and disease-causing single-nucleotide polymorphisms via comparative nucleotide sequence analyses, and thereby predict potential functions and/or disease implications of identified ncRNAs. ${ }^{122}$ Similar efforts to move from pure identification of ncRNAs to functional interpretations of their mechanisms of action will be critical, particularly as whole-transcriptome RNA-seq becomes more prevalent.

In summary, the transcriptome of the developing human brain represents a nexus of genetic and environmental interaction that has only recently begun to be rigorously mapped. In particular, it has become clear that non-coding regions of the genome and 
gene-gene interactions encode layers of information that are of unique importance to human development, to brain development and non-additively to the developing human brain in particular. Although this field is still emerging, already there is evidence that alterations in gene network and ncRNAs may partially underlie common neurodevelopmental disorders. As more studies of gene and ncRNA expression are performed in human post-mortem brain tissue and improving model systems, this area of research is likely to help explain how genes and the environment interact to shape brain development.

\section{CONFLICT OF INTEREST}

The authors declare no conflict of interest.

\section{ACKNOWLEDGMENTS}

The Intramural Research Program at the National Institute of Child Health and Human Development supported this work. MNZ was also supported by Baylor College of Medicine MSTP and the NIH-University of Cambridge Biomedical Scholars Program. LPG was funded by the National Institute of Mental Health. The funders had no role in study design, data collection and analysis, decision to publish or preparation of the manuscript. We apologize for unintentionally omitting other relevant work because of space limitations. We would like to thank Jeremy Swan for help with figure generation.

\section{REFERENCES}

1 Enard W, Khaitovich P, Klose J, Zöllner S, Heissig F, Giavalisco P et al. Intra- and interspecific variation in primate gene expression patterns. Science 2002; 296: 340-343.

2 Lockhart DJ, Barlow C. DNA arrays and gene expression analysis in the brain. In: Chin HR, Moldin SO (eds). Methods in Genomic Neuroscience. New York, NY, USA CRC Press, 2001, pp 109-140.

3 Caceres M, Lachuer J, Zapala MA, Redmond JC, Kudo L, Geschwind DH et al Elevated gene expression levels distinguish human from non-human primate brains. Proc Natl Acad Sci USA 2003; 100: 13030-13035.

4 Khaitovich P, Muetzel B, She X, Lachmann M, Hellmann I, Dietzsch J. Regional patterns of gene expression in human and chimpanzee brains. Genome Res 2004; 14: 1462-1473.

5 Kang HJ, Kawasawa Yl, Cheng F, Zhu Y, Xu X, Li M et al. Spatio-temporal transcriptome of the human brain. Nature 2011; 478: 483-489.

6 de la Grange P, Gratadou L, Delord M, Dutertre M, Auboeuf D. Splicing factor and exon profiling across human tissues. Nucleic Acids Res 2010; 38: 2825-2838.

7 Ramskold D, Wang ET, Burge CB, Sandberg R. An abundance of ubiquitously expressed genes revealed by tissue transcriptome sequence data. PLoS Comput Biol 2009; 5: e1000598.

8 Howald C, Tanzer A, Chrast J, Kokocinski F, Derrien T, Walters N et al. Combining RT-PCR-seq and RNA-seq to catalog all genic elements encoded in the human genome. Genome Res 2012; 9: 1698-1710.

9 Soumillon M, Necsulea A, Weier M, Brawand D, Zhang X, Gu H et al. Cellular source and mechanisms of high transcriptome complexity in the mammalian testis. Cell Rep 2013; 3: 2179-2190.

10 Yeo G, Holste D, Kreiman G, Burge CB. Variation in alternative splicing across human tissues. Genome Biol 2004; 5: R74.

11 Wang ET, Sandberg R, Luo S, Khrebtukova I, Zhang L, Mayr C et al. Alternative isoform regulation in human tissue transcriptomes. Nature 2008; 456: 470-476.

12 Mortazavi A, Williams BA, McCue K, Schaeffer L, Wold B. Mapping and quantifying mammalian transcriptomes by RNA-Seq. Nat Methods 2008; 5: 621-628.

13 Qureshi IA, Mehler MF. Emerging roles of non-coding RNAs in brain evolution, development, plasticity and disease. Nat Rev Neurosci 2012; 13: 528-541.

14 Chodroff RA, Goodstadt L, Sirey TM, Oliver PL, Davies KE, Green ED et al. Long noncoding RNA genes: Conservation of sequence and brain expression among diverse amniotes. Genome Biol 2010; 11: R72

15 Kuss AW, Chen W. MicroRNAs in brain function and disease. Curr Neurol Neurosc Rep 2008; 8: 190-197.

16 Ponjavic J, Oliver PL, Lunter G, Ponting CP. Genomic and transcriptional co-localization of protein-coding and long non-coding RNA pairs in the developing brain. PLoS Genet 2009; 5: e1000617.

17 Schonrock N, Ke YD, Humphreys D, Staufenbiel M, Ittner LM, Preiss T et al. Neuronal microRNA deregulation in response to Alzheimer's disease amyloid-beta. PLoS One 2010; 5: e11070.
18 St Laurent G, Faghihi MA, Wahlestedt C. Non-coding RNA transcripts: Sensors of neuronal stress, modulators of synaptic plasticity, and agents of change in the onset of Alzheimer's disease. Neurosci Lett 2009; 466: 81-88.

19 Qureshi IA, Mehler MF. Non-coding RNA networks underlying cognitive disorders across the lifespan. Trends Mol Med 2011; 17: 337-346.

20 Geschwind D, Levitt P. Autism spectrum disorders: developmental disconnection syndromes. Curr Opin Neurobiol 2007; 17: 103-111.

21 Nadler JJ, Zou F, Huang H, Moy SS, Lauder J, Crawley JN et al. Large-scale gene expression differences across brain regions and inbred strains correlate with a behavioral phenotype. Genetics 2006; 174: 1229-1336.

22 Roth RB, Hevezi P, Lee J, Willhite D, Lechner SM, Foster AC et al. Gene expression analyses reveal molecular relationships among 20 regions of the human CNS Neurogenetics 2006; 7: 67-80.

23 Strand AD, Aragaki AK, Baquet ZC, Hodges A, Cunningham P, Holmans $P$ et al. Conservation of regional gene expression in mouse and human brain. PLoS Genet 2007; 3: e59.

24 Fatemi SH, Aldinger KA, Ashwood $\mathrm{P}$, Bauman ML, Blaha CD, Blatt GJ et al. Consensus paper: pathological role of the cerebellum in autism. Cerebellum 2012; 11: 777-807.

25 Molyneaux BJ, Arlotta P, Menezes JR, Macklis JD. Neuronal subtype specification in the cerebral cortex. Nat Rev Neurosci 2007; 8: 427-437.

26 Naumova OY, Palejev D, Vlasova NV, Lee M, Rychkov SY, Babich ON et al. Agerelated changes of gene expression in the neocortex: preliminary data on RNAseq of the transcriptome in three functionally distinct cortical areas. Dev Psychopathol 2008; 24: 1427-1442.

27 Dekaban AS, Sadowsky D. Changes in brain weights during the span of human life: relation of brain weights to body heights and body weights. Ann Neurol 1978; 4: 345-356.

28 Sowell ER, Thompson PM, Toga AW. Mapping changes in the human cortex throughout the span of life. Neuroscientist 2004; 10: 372-392.

29 Johnson MB, Kawasawa Yl, Mason CE, Krsnik Z, Coppola G, Bogdanovic D et al. Functional and evolutionary insights into human brain development through global transcriptome analysis. Neuron 2009; 62: 494-509.

30 Lambert N, Lambot MA, Bilheu A, Albert V, Englert Y, Libert F et al. Genes expressed in specific areas of the human fetal cerebral cortex display distinct patterns of evolution. PLoS One 2011; 6: e17753.

31 Colantuoni C, Lipska BK, Ye T, Hyde TM, Tao R, Leek JT et al. Temporal dynamics and genetic control of transcription in the human prefrontal cortex. Nature 2011 478: $519-524$

32 Somel M, Franz H, Yan Z, Lorenc A, Guo S, Giger T et al. Transcriptional neoteny in the human brain. Proc Natl Acad Sci USA 2009; 106: 5743-5757.

33 Somel M, Guo S, Fu N, Yan Z, Hu HY, Xu Y et al. MicroRNA, mRNA, and protein expression link development and aging in human and macaque brain. Genome Res 2010; 20: 1207-1218.

34 Ponting $\mathrm{CP}$, Belgard TG. Transcribed dark matter: meaning or myth? Hum Mol Genet 2010; 19: R162-R168.

35 Ponting CP, Oliver PL, Reik W. Evolution and functions of long noncoding RNAs. Cell 2009; 136: 629-641.

36 Mercer TR, Mattick JS. Structure and function of long noncoding RNAs in epigenetic regulation. Nat Struct Mol Biol 2013; 20: 300-307.

37 Hansen TB, Jensen TI, Clausen BH, Bramsen JB, Finsen B, Damgaard CK et al. Natural RNA circles function as efficient microRNA sponges. Nature 2013; 495: 384-388.

38 Mansfield KD, Keene JD. The ribonome: a dominant force in co-ordinating gene expression. Biol Cell 2009; 101: 169-181.

39 Skog J, Würdinger T, van Rijn S, Meijer DH, Gainche L, Sena-Esteves M et al. Glioblastoma microvesicles transport RNA and proteins that promote tumour growth and provide diagnostic biomarkers. Nat Cell Biol 2008; 10: 1470-1476.

40 Balaj L, Lessard R, Dai L, Cho YJ, Pomeroy SL, Breakefield XO et al. Tumour microvesicles contain retrotransposon elements and amplified oncogene sequences. Nat Commun 2011; 2: 180.

41 Mercer TR, Dinger ME, Sunkin SM, Mehler MF, Mattick JS. Specific expression of long noncoding RNAs in the mouse brain. Proc Natl Acad Sci USA 2008; 105: 716-721.

42 Cabili MN, Trapnell C, Goff L, Koziol M, Tazon-Vega B, Regev A et al. Integrative annotation of human large intergenic noncoding RNAs reveals global properties and specific subclasses. Genes Dev 2011; 25: 1915-1927.

43 Derrien T, Johnson R, Bussotti G, Tanzer A, Djebali S, Tilgner H et al. The GENCODE v7 catalog of human long noncoding RNAs: analysis of their gene structure, evolution, and expression. Genome Res 2012; 22: 1775-1789.

44 Pollard KS, Salama SR, Lambert N, Lambot MA, Coppens S, Pedersen JS et al. An RNA gene expressed during cortical development evolved rapidly in humans. Nature 2006; 443: 167-172.

45 Paz-Yaacov N, Levanon EY, Nevo E, Kinar Y, Harmelin A, Jacob-Hirsch J et al. Adenosine-to-inosine RNA editing shapes transcriptome diversity in primates. Proc Natl Acad Sci USA 2010; 107: 12174-12179. 
46 Hutvagner G, Zamore PD. A microRNA in a multiple-turnover RNAi enzyme complex. Science 2002; 297: 2056-2060.

47 Liu Q, Paroo Z. Biochemical principles of small RNA pathways. Annu Rev Biochem 2010; 79: 295-319.

48 Hashimoto Y, Akiyama Y, Yuasa Y. Multiple-to-multiple relationships between microRNAs and target genes in gastric cancer. PLoS One 2013; 8: e62589.

49 Hansen TB, Wiklund ED, Bramsen JB, Villadsen SB, Statham AL, Clark SJ et al. miRNA-dependent gene silencing involving Ago2-mediated cleavage of a circular antisense RNA. EMBO J 2011; 30: 4414-4422.

50 Somel M, Liu X, Tang L, Yan Z, Hu H, Guo S et al. MicroRNA-driven developmental remodeling in the brain distinguishes humans from other primates. PLoS Biol 2011; 9: e1001214.

$51 \mathrm{Hu}$ HY, Guo S, Xi J, Yan Z, Fu N, Zhang X et al. MicroRNA expression and regulation in human, chimpanzee, and macaque brains. PLoS Genet 2011; 7: e1002327.

52 Giraldez AJ, Cinalli RM, Glasner ME, Enright AJ, Thomson JM, Baskerville S et al. MicroRNAs regulate brain morphogenesis in zebrafish. Science 2005; 308 : 833-838.

53 Davis TH, Cuellar TL, Koch SM, Barker AJ, Harfe BD, McManus MT et al. Conditional loss of Dicer disrupts cellular and tissue morphogenesis in the cortex and hippocampus. J Neurosci 2008; 28: 4322-4330.

54 Zhao X, He X, Han X, Yu Y, Ye F, Chen Y et al. MicroRNA-mediated control of oligodendrocyte differentiation. Neuron 2010; 65: 612-626.

55 Zhao C, Sun G, Li S, Shi Y. A feedback regulatory loop involving microRNA-9 and nuclear receptor TLX in neural stem cell fate determination. Nat Struct Mol Biol 2009; 16: 365-371.

56 Shibata M, Nakao H, Kiyonari H, Abe T, Aizawa S. MicroRNA-9 regulates neurogenesis in mouse telencephalon by targeting multiple transcription factors. J Neurosci 2011; 31: 3407-3422.

57 Yoo AS, Sun AX, Li L, Shcheglovitov A, Portmann T, Li Y et al. MicroRNA-mediated conversion of human fibroblasts to neurons. Nature 2011; 476: 228-231.

$58 \mathrm{Wu}$ J, Xie X. Comparative sequence analysis reveals an intricate network among REST, CREB and miRNA in mediating neuronal gene expression. Genome Biol 2006; 7: R85.

59 Dugas JC, Cuellar TL, Scholze A, Ason B, Ibrahim A, Emery B et al. Dicer1 and miR-219 are required for normal oligodendrocyte differentiation and myelination. Neuron 2010; 65: 597-611.

60 Tao J, Wu H, Lin Q, Wei W, Lu XH, Cantle JP et al. Deletion of astroglial Dicer causes non-cell-autonomous neuronal dysfunction and degeneration. $J$ Neurosci 2011; 31: 8306-8319.

61 Natera-Naranjo O, Aschrafi A, Gioio AE, Kaplan BB. Identification and quantitative analyses of microRNAs located in the distal axons of sympathetic neurons. RNA 2010; 16: 1516-1529.

62 Hansen KF, Karelina K, Sakamoto K, Wayman GA, Impey S, Obrietan K. miRNA-132: a dynamic regulator of cognitive capacity. Brain Struct Funct 2013; 218: 817-831.

63 Smibert P, Bejarano F, Wang D, Garaulet DL, Yang JS, Martin R et al. A Drosophila genetic screen yields allelic series of core microRNA biogenesis factors and reveals post-developmental roles for microRNAs. RNA 2011; 17: 1997-2010.

64 Schofield CM, Hsu R, Barker AJ, Gertz CC, Blelloch R, Ullian EM. Monoallelic deletion of the microRNA biogenesis gene Dgcr8 produces deficits in the development of excitatory synaptic transmission in the prefrontal cortex. Neural Dev 2011; 6: 11.

65 Konopka W, Kiryk A, Novak M, Herwerth M, Parkitna JR, Wawrzyniak M et al. MicroRNA loss enhances learning and memory in mice. J Neurosci 2010; 30: 14835-14842.

66 Liu C, Zhang F, Li T, Lu M, Wang L, Yue W et al. MirSNP, a database of polymorphisms altering miRNA target sites, identifies miRNA-related SNPs in GWAS SNPs and eQTLs. BMC Genomics 2012; 13: 661

67 Silber J, Lim DA, Petritsch C, Persson Al, Maunakea AK, Yu M et al. miR-124 and miR-137 inhibit proliferation of glioblastoma multiforme cells and induce differentiation of brain tumor stem cells. BMC Med 2008; 6: 14

68 Martins M, Rosa A, Guedes LC, Fonseca BV, Gotovac K, Violante S et al. Convergence of miRNA expression profiling, a-synuclein interacton and GWAS in Parkinson's disease. PLoS One 2011; 6: e25443.

69 Abelson JF, Kwan KY, O'Roak BJ, Baek DY, Stillman AA, Morgan TM et al. Sequence variants in SLITRK1 are associated with Tourette's syndrome. Science 2005; 310: 317-320.

70 Ziats MN, Rennert OM. Identification of differentially expressed microRNAs across the developing human brain. Mol Psychiatry 2014; 19: 848-852.

71 Carninci P, Kasukawa T, Katayama S, Gough J, Frith MC, Maeda N et al. The transcriptional landscape of the mammalian genome. Science 2005; 309: 1559-1563.

72 Djebali S, Davis CA, Merkel A, Dobin A, Lassmann T, Mortazavi A et al. Landscape of transcription in human cells. Nature 2012; 489: 101-108.
73 Amaral PP, Mattick JS. Noncoding RNA in development. Mamm Genome 2008; 19: 454-492.

74 Lipovich L, Dachet F, Cai J, Bagla S, Balan K, Jia H et al. Activity-dependent human brain coding/noncoding gene regulatory networks. Genetics 2012; 192: 1133-1148.

75 Bernard D, Prasanth KV, Tripathi V, Colasse S, Nakamura T, Xuan Z et al. A long nuclear-retained non-coding RNA regulates synaptogenesis by modulating gene expression. EMBO J 2010; 29: 3082-3093.

76 Bond AM, Vangompel MJ, Sametsky EA, Clark MF, Savage JC, Disterhoft JF et al. Balanced gene regulation by an embryonic brain ncRNA is critical for adult hippocampal GABA circuitry. Nat Neurosci 2009; 12: 1020-1027.

77 Belgard TG, Marques AC, Oliver PL, Abaan HO, Sirey TM, Hoerder-Suabedissen A et al. A transcriptomic atlas of mouse neocortical layers. Neuron 2011; 71: 605-616.

78 Barry G, Briggs JA, Vanichkina DP, Poth EM, Beveridge NJ, Ratnu VS et al. The long non-coding RNA Gomafu is acutely regulated in response to neuronal activation and involved in schizophrenia-associated alternative splicing. Mol Psychiatry 2014; 19: 486-494.

79 Faghihi MA, Modarresi F, Khalil AM, Wood DE, Sahagan BG, Morgan TE et al. Expression of a noncoding RNA is elevated in Alzheimer's disease and drives rapid feed-forward regulation of beta-secretase. Nat Med 2008; 14: 723-730.

80 Modarresi F, Faghihi MA, Patel NS, Sahagan BG, Wahlestedt C, Lopez-Toledano MA. Knockdown of BACE1-AS nonprotein-coding transcript modulates betaamyloid-related hippocampal neurogenesis. Int J Alzheimers Dis 2011; 2011: 929042.

81 Landers M, Calciano MA, Colosi D, Glatt-Deeley H, Wagstaff J, Lalande M. Maternal disruption of Ube3a leads to increased expression of Ube3a-ATS in trans. Nucleic Acids Res 2005; 33: 3976-3984.

82 Meng L, Person RE, Beaudet AL. Ube3a-ATS is an atypical RNA polymerase II transcript that represses the paternal expression of Ube3a. Hum Mol Genet 2012; 21: $3001-3012$.

83 Sharan R, Suthram S, Kelley RM, Kuhn T, McCuine S, Uetz P et al. Conserved patterns of protein interaction in multiple species. Proc Natl Acad Sci USA 2005; 102: 1974-1979.

84 Sanchez-Vives MV, McCormick DA. Cellular and network mechanisms of rhythmic recurrent activity in neocortex. Nat Neurosci 2000; 3: 1027-1034.

85 Bullmore E, Sporns O. Complex brain networks: graph theoretical analysis of structural and functional systems. Nat Rev Neurosci 2009; 10: 186-198.

86 Croft DP, Krause J, James R. Social networks in the guppy (Poecilia reticulata). Proc Biol Sci 2004; 271: S516-S519.

87 Hajeri VA, Amatruda JF. Studying synthetic lethal interactions in the zebrafish system: insight into disease genes and mechanisms. Dis Model Mech 2012; 5: 33-37.

88 Phillips PC. Epistasis--the essential role of gene interactions in the structure and evolution of genetic systems. Nat Rev Genet 2008; 9: 855-867.

89 Stuart J, Segal E, Koller D, Kim S. A gene-coexpression network for global discovery of conserved genetic modules. Science 2003; 302: 249-255.

90 Wolfe C, Kohane I, Butte A. Systematic survey reveals general applicability of "guilt-by-association" within gene coexpression networks. BMC Bioinformatics 2005; 6: 227.

91 Oldham M, Konopka G, Iwamoto K, Langfelder P, Kato T, Horvath S et al. Functional organization of the transcriptome in human brain. Nat Neurosci 2008; 11: $1271-1282$

92 Winden K, Oldham M, Mirnics K, Ebert P, Swan C, Levitt P et al. The organization of the transcriptional network in specific neuronal classes. Mol Syst Biol 2009; 5: 291.

93 Pérez-Bercoff $\AA$, Hudson CM, Conant GC. A conserved mammalian protein interaction network. PLoS One 2013; 8: e52581.

94 Baryshnikova A, Costanzo M, Kim Y, Ding H, Koh J, Toufighi K et al. Quantitative analysis of fitness and genetic interactions in yeast on a genome scale. Nat Methods 2010; 7: 1017-1024.

95 Voineagu I. Gene expression studies in autism: moving from the genome to the transcriptome and beyond. Neurobiol Dis 2012; 45: 69-75.

96 Cookson W, Liang L, Abecasis G, Moffatt M, Lathrop M. Mapping complex disease traits with global gene expression. Nat Rev Genet 2009; 10: 184-194.

97 Purcell $\mathrm{AE}$, Jeon $\mathrm{OH}$, Zimmerman AW, Blue ME, Pevsner J. Postmortem brain abnormalities of the glutamate neurotransmitter system in autism. Neurology 2001; 57: 1618-1628.

98 Garbett K, Ebert PJ, Mitchell A, Lintas C, Manzi B, Mirnics K et al. Immune transcriptome alterations in the temporal cortex of subjects with autism. Neurobiol Dis 2008; 30: 303-311.

99 Voineagu I, Wang X, Johnston P, Lowe JK, Tian Y, Horvath S et al. Transcriptomic analysis of autistic brain reveals convergent molecular pathology. Nature 2011; 474: 380-384.

100 Chow ML, Pramparo T, Winn ME, Barnes CC, Li HR, Weiss L et al. Age-dependent brain gene expression and copy number anomalies in autism suggest distinct pathological processes at young versus mature ages. PLoS Genet 2012; 8: e1002592. 
101 Ginsberg MR, Rubin RA, Falcone T, Ting AH, Natowicz MR. Brain transcriptional and epigenetic associations with autism. PLoS One 2012; 7: e44736.

102 Walker S, Scherer SW. Identification of candidate intergenic risk loci in autism spectrum disorder. BMC Genomics 2013; 14: 499.

103 Abu-Elneel K, Liu T, Gazzaniga FS, Nishimura Y, Wall DP, Geschwind DH et al. Heterogeneous dysregulation of microRNAs across the autism spectrum. Neurogenetics 2008; 9: 153-161.

104 Talebizadeh Z, Butler MG, Theodoro MF. Feasibility and relevance of examining lymphoblastoid cell lines to study role of microRNAs in autism. Autism Res 2008 1: 240-250.

105 Sarachana T, Zhou R, Chen G, Manji HK, Hu VW. Investigation of posttranscriptional gene regulatory networks associated with autism spectrum disorders by microRNA expression profiling of lymphoblastoid cell lines. Genome Med 2010; 2: 23.

106 Ghahramani Seno MM, Hu P, Gwadry FG, Pinto D, Marshall CR, Casallo G et al. Gene and miRNA expression profiles in autism spectrum disorders. Brain Res 2011; 1380: 85-97.

107 Velmeshev D, Magistri M, Faghihi MA. Expression of non-protein-coding antisense RNAs in genomic regions related to autism spectrum disorders. Mol Autism 2013; 4: 32.

108 Ziats MN, Rennert OM. Aberrant expression of long noncoding RNAs in autistic brain. J Mol Neurosci 2013; 49: 589-593.

109 Kerin T, Ramanathan A, Rivas K, Grepo N, Coetzee GA, Campbell DB. A noncoding RNA antisense to moesin at 5p14.1 in autism. Sci Transl Med 2012; 4 $128 \mathrm{ra} 40$.

110 O'Roak BJ, Vives L, Girirajan S, Karakoc E, Krumm N, Coe BP et al. Sporadic autism exomes reveal a highly interconnected protein network of de novo mutations. Nature 2012; 485: 246-250.

111 Gilman SR, lossifov I, Levy D, Ronemus M, Wigler M, Vitkup D. Rare de novo variants associated with autism implicate a large functional network of genes involved in formation and function of synapses. Neuron 2011; 70: 898-907.

112 Ben-David E, Shifman S. Networks of neuronal genes affected by common and rare variants in autism spectrum disorders. PLoS Genet 2012; 8: e1002556.

113 Ben-David E, Shifman S. Combined analysis of exome sequencing points toward a major role for transcription regulation during brain development in autism. Mol Psychiatry 2013; 18: 1054-1056.
114 Willsey AJ, Sanders SJ, Li M, Dong S, Tebbenkamp AT, Muhle RA et al. Coexpression networks implicate human midfetal deep cortical projection neurons in the pathogenesis of autism. Cell 2013; 155: 997-1007.

115 Parikshak NN, Luo R, Zhang A, Won H, Lowe JK, Chandran V et al. Integrative functional genomic analyses implicate specific molecular pathways and circuits in autism. Cell 2013; 155: 1008-1021.

116 Abbott A. Tissue-bank shortage: brain child. Nature 2011; 478: 442-443.

117 Stein JL, de la Torre-Ubieta L, Tian Y, Parikshak NN, Hernández IA, Marchetto MC et al. A quantitative framework to evaluate modeling of cortical development by neural stem cells. Neuron 2014; 83: 69-86.

118 Germain ND, Chen PF, Plocik AM, Glatt-Deeley H, Brown J, Fink JJ et al. Gene expression analysis of human induced pluripotent stem cell-derived neurons carrying copy number variants of chromosome 15q11-q13.1. Mol Autism 2014; 5: 1-19.

119 Medvedev SP, Shevchenko Al, Zakian SM. Induced pluripotent stem cells: problems and advantages when applying them in regenerative medicine. Acta Naturae 2010; 2: 18-28.

120 Okita K, Yamanaka S. Induced pluripotent stem cells: opportunities and challenges. Philos Trans R Soc Lond B Biol Sci 2011; 366: 2198-2207.

121 Lancaster MA, Renner M, Martin C, Wenzel D, Bicknell LS, Hurles ME et al. Cerebral organoids model human brain development and microcephaly. Nature 2013; 501: 374-379.

122 Seetin MG, Mathews DH. RNA structure prediction: an overview of methods. Methods Mol Biol 2012; 905: 99-122.

123 Krumm N, O'Roak BJ, Shendure J, Eichler EE. A de novo convergence of autism genetics and molecular neuroscience. Trends Neurosci 2014; 37 95-105.

This work is licensed under a Creative Commons Attribution 4.0 International License. The images or other third party material in this article are included in the article's Creative Commons license, unless indicated otherwise in the credit line; if the material is not included under the Creative Commons license, users will need to obtain permission from the license holder to reproduce the material. To view a copy of this license, visit http://creativecommons.org/licenses/ by/4.0/ 